\title{
Diagnosing Antiphospholipid Antibody Syndrome: A Review of the Criterion for Definite APS
}

\author{
Jeffrey S. Dlott
}

Quest Diagnostics Nichols Institute, 14225 Newbrook Dr., Chantilly, Virginia 20151, USA

\begin{abstract}
Antiphospholipid Antibody Syndrome (APS) is a highly prevalent cause of antibody-mediated thrombosis manifesting in venous thrombosis (DVT and PE), arterial thrombosis (most commonly stroke), and pregnancy complications. The diagnosis of definite APS requires both clinical and laboratory criterion as established by the working group of the International Congress on Antiphospholipid Antibodies (based on expert opinion). Since thrombosis and pregnancy loss are common in the general population, and antiphospholipid antibodies (aPL) occurs in a small percentage of the healthy public, it is important to demonstrate antibody persistence in patients who have the proper clinical indications in order to avoid misdiagnosis. Unfortunately, laboratory testing in this area lacks standardization, resulting in wide inter-laboratory variance. However, due to the commercialization of tests and automation, inter-laboratory variance has improved. Data on several new non-criterion tests suggest that they may improve the specificity or risk stratification for thrombosis. A new guidance document on aPL testing strives to achieve better consistency, but much work remains to be done in the area of standardization.
\end{abstract}

Keywords: Antibody Mediated thrombosis, antiphospholipid antibody syndrome, cardiolipin, beta 2 GPI, lupus anticoagulant, thrombosis.

\section{INTRODUCTION}

In the early 1980's, Graham Hughes and colleagues described the association between antibodies against phospholipid (aPL) and thrombosis, spontaneous abortion, neurologic disease, thrombocytopenia, pulmonary hypertension, and livedo reticularis [1]. This disease was originally named the anticardiolipin syndrome and then primary antiphospholipid syndrome (APS), APS differs from classic SLE in that many affected patients lack features of underlying connective tissue diseases (CTD) [1]. The International Congress on Antiphospholipid Antibodies (aPL) first convened in London, UK, in 1984, reconvening every 2-3 years thereafter. A major accomplishment of the congresses has been the establishment of classification criteria for APS. While the original intent of the classification criteria to compare and risk stratify patients in clinical studies, they have been adopted as diagnostic criteria in routine clinical practice. In 1998 the Congress working in Sapporo, Japan established the first diagnostic criteria (Sapporo Criteria) for "definite" APS which were then updated in 2006 (Sidney Criteria) [2].

The term secondary APS has been used in the literature to describe patients with APS who also have other autoimmune disease, usually SLE. However, the clinical consequences of aPL appear to be similar for patients in these two categories. It is unclear whether the pathogenesis of APS and secondary APS results from two distinct

*Address correspondence to this author at the Quest Diagnostics Nichols Institute, 14225 Newbrook Dr., Chantilly, Virginia 20151, USA;

Tel: 703-802-7259; Fax: 703-802-7099;

E-mail: Jeffrey.s.dlott@questdiagnostics.com processes, a predisposition to develop additional antibodies, or if APS and SLE are part of the same spectrum. For purposes of classification, it is recommended that "SLE" or "other CTD" be documented rather than APS [2]. Similarly, patients should be recognized for the presence or absence of additional risk factors for thrombosis (i.e. established risk factors for cardiovascular disease, inherited thrombophilia, oral contraceptives, nephrotic syndrome, malignancy, immobilization, and surgery) [2].

APS is now defined as an autoimmune disorder associated with arterial or venous thrombosis, recurrent pregnancy loss, and persistently positive aPL. Within this framework, the clinical and laboratory criteria are intended to improve the specificity for the diagnosis of definite APS (Table 1) [2]. The clinical and laboratory associations recognized as associated with APS but not included in the criteria are referred to collectively as "non-criteria" features [2].

\section{CLINICAL FEATURES}

Thrombosis may occur in arterial or venous vasculature in any tissue or organ. Objective evidence such as imaging studies or histopathology is required for diagnosis. Significant inflammation of the vessel wall should be excluded to prevent misclassification of a vasculitis as APS. A remote thrombotic event could be considered as a clinical criterion if it was objectively documented. APS accounts for approximately $10 \%$ of acute venous thromboembolic disease (VTE) [3]. Deep vein thrombosis (DVT) of the lower extremities is the most common presentation with nearly half of patients having pulmonary embolism (PE) as well [3]. Superficial vein thrombosis is not included in the clinical 
Table 1. Diagnostic criteria [2].

\begin{tabular}{|l|l|}
\hline \multicolumn{1}{|c|}{ Clinical Criteria } & \multicolumn{1}{|c|}{ Laboratory Criteria } \\
\hline \hline $\begin{array}{l}\text { Vascular thrombosis - one or more episodes of arterial, venous or small } \\
\text { vessel thrombosis in any tissue or organ (confirmed by imaging or } \\
\text { histopathology) }\end{array}$ & Lupus anticoagulant in plasma on two occasions at least 12 weeks apart \\
\hline $\begin{array}{l}\text { Recurrent pregnancy loss (after }>10 \text { weeks' gestation, or 3 after }<10 \text { weeks' } \\
\text { gestation) or one or more premature births due to pregnancy complications }\end{array}$ & $\begin{array}{l}\text { Anticardiolipin antibodies of IgG and/or IgM isotype on two occasions at } \\
\text { least 12 weeks apart }\end{array}$ \\
\hline & $\begin{array}{l}\text { Anti- } \beta 2 \text {-GPI antibody of IgG or IgM isotype on two occasions at least } 12 \\
\text { weeks apart }\end{array}$ \\
\hline Definite APS is considered to be present if at least one of the clinical and one of the laboratory criteria are met. \\
\hline $\begin{array}{l}\text { Ig, immunoglobulin; GPI, glycoprotein-I. } \\
\text { Antiphospholipid syndrome is considered to definitely present when at least one clinical criterion and one laboratory criterion are met. }\end{array}$
\end{tabular}

criteria [2]. The most frequent site for arterial thrombosis in patients with APS is the cerebral vasculature resulting in transient ischemic attacks stroke, or both [4]. In patients $<45$ years old, more than $20 \%$ of the strokes are associated with APS [5].

Non-criteria features include livedo reticularis, cardiac valve disease, thrombocytopenia, and nephropathy [2]. For each of the aforementioned, aPL-associated definitions exist [2]. The expert committee in Sydney considered the adoption of these associations as independent criteria, but felt that they decreased the specificity for definite APS [2].

Approximately $10-15 \%$ of women with recurrent miscarriage are diagnosed with APS [6]. Pregnancy associated morbidity is challenging because no widely accepted definition of placental insufficiency or characteristic histopathologic placental abnormality in APS exists. Therefore, to avoid misclassification, experts recommend strict adherence to standard clinical definitions of eclampsia, severe preeclampsia, and placental insufficiency [2]. Non-criteria obstetric morbidity associated with APS includes placental abruption, late premature birth, two unexplained miscarriages, and two or more unexplained in vitro fertilization failures $[7,8]$.

Catastrophic APS (CAPS) is a variant that occurs in less than $1 \%$ of patients with APS. It is characterized by sudden extensive microvascular thrombosis in three or more organs leading to multi-organ failure [9-11]. Mortality is very high (approaching 50\%) [11].

aPL may be detected in $1-3.5 \%$ of the healthy population and associated with, systemic autoimmune diseases, certain drugs (i.e. antipsychotic, antiarrhythmic), infection (i.e. HCV, HIV, VZV, syphilis), malignancy, and advancing age $[12,13]$. This may be due to neo or cryptic epitope expression, or molecular mimicry [14]. In addition, thrombosis, pregnancy loss, and transient aPL positivity are common. Therefore, the pretest probability of APS is improved by testing patients with specific clinical indications (Table 2) [4, 15].

\section{LABORATORY EVALUATION}

aPL describes a heterogeneous group of antibodies against anionic phospholipids or protein complexed phospholipids. Assays used in the evaluations are either solid phase (immobilized antigen) or liquid (clot based). Cardiolipin is the most common phospholipid substrate. Beta-2 Glycoprotein $\left(\beta_{2} \mathrm{GPI}\right)$ and Prothrombin are the most common phospholipid bound proteins inhibitors in APS. Lupus anticoagulants are immunoglobulins (IgG, IgM/mixture), which interfere with one or more of the in vitro phospholipid (PL) dependent steps of coagulation, resulting in prolonged coagulation tests (i.e. aPTT, or dilute Russell Viper Venom Time [dRVVT]).

Table 2. Indications for antiphospholipid antibodies testing.

\begin{tabular}{|ll|}
\hline & Indication for Antiphospholipid Antibody Testing \\
\hline \hline Consider testing in all patient with: \\
\hline$\bullet$ & Unprovoked proximal DVT or PE especially in the young or in those with thrombosis at unusual sites \\
\hline$\bullet$ & Recurrent thrombosis \\
\hline$\bullet$ & Young adults ( $<50$ years) with ischaemic stroke \\
\hline & Systemic lupus erythematous or those with autoimmune disease and thrombosis \\
\hline & Recurrent pregnancy loss or pregnancy complication with premature birth \\
\hline & Unexplained thrombocytopenia \\
\hline & Livedo reticularis \\
\hline & Thrombosis is an unusual site: retinal vein, portal, cerebral venous sinus, renal vein \\
\hline
\end{tabular}

DVT, deep vein thrombosis; PE, pulmonary embolism. 


\section{ANTICARDIOLIPIN/ANTI-BETA-2 GLYCOPROTEIN ANTIBODIES}

Anticardiolipin (aCL) and anti $\beta_{2}$ GPI antibodies are usually assessed using solid phase assays. The most common platform is the enzyme-linked immunosorbent assay (ELISA), although fully automated chemiluminescent assays have been recently introduced. Cardiolipin or $\beta_{2} \mathrm{GPI}$ is immobilized on the bottom of a well on a polystyrene plate to which the immunologic reaction with patient sample (serum or citrated platelet poor plasma) occurs. For aCL assays human $\beta_{2} \mathrm{GPI}$ is recommended as an additional reagent, thus transforming the assay into a $\beta_{2} \mathrm{GPI}$-dependent $\mathrm{aCL}$ and a more specific assay [16]. Since both assays use a source of human $\beta_{2}$ GPI, a high correlation between modern aCL $\beta_{2}$ GPI assays is often observed. Indeed, aCL and $\beta_{2} \mathrm{GPI}$ positivity of the same isotype reinforces the probability of APS.

Medium and high titers of aCL are associated with the clinical manifestation of APS. Use of Secondary standards (i.e. Harris Standards), are often used to establish thresholds for positivity. They are made by mixing varying quantities of high positive with normal sera, and expressed as IgG or IgM antiphospholipid units (GPL or MPL). The Sapporo criteria established a threshold of $>40 \mathrm{MPL}$ or GPL as the laboratory criteria for medium aCL positivity [17]. Alternatively a threshold of $>99^{\text {th }}$ percentile of normal controls may be used as the cutoff point for positivity for both aCL and anti- $\beta_{2} \mathrm{GPI}$ antibodies [2]. In contrast to aCL, anti- $\beta_{2}$ GPI antibodies are expressed in arbitrary units. Persistent positivity of aPL is an important criterion for definite APS since transient aPL is not infrequent in clinical practice. Per expert opinion outlined in the Sydney criteria, a time interval of at least 12 weeks and no more than 5 years must occur between positive tests [2].

No gold standards or international standardization of aPL testing exists, because aPL assays may vary in performance characteristics, interferences, standards and calibrators, quality control, result expression, cut-off values and result interpretation. Consequently inter-laboratory results vary greatly, whereas intra-laboratory results are more consistent. False positive results may be seen with rheumatoid factor (RF), heterophile antibodies, human anti-animal antibodies, high levels of monoclonal immunoglobulin, and cryoglobulins, particularly with IgM aCL [18]. The association of false positive RPRs (reagent that contains cardiolipin) and positive aCL by ELISA was described by Harris et al. [19]. The frequency of this association was examined in a large nationwide series of pregnant women in which the authors reported a titer dependent association in $1 / 3$ to half of the patients studied [20].

\section{LUPUS ANTICOAGULANT}

Diagnosing a lupus anticoagulant requires four criteria: (1) an abnormal clot-based screening test (preferably with dilute phospholipid); (2) demonstration of an inhibitor on mixing study, (3) demonstration that the inhibitor is PL dependent (confirmatory test); and (4) exclusion of other coagulopathies that could give false positive LA results (i.e. specific Factor inhibitors or high dose anticoagulant therapy) $[21,22]$. Weak LA may be difficult to diagnose as results typically border the cut-offs, however, weak LA should be considered positive if the clinical scenario is appropriate and other causes of clot have been excluded [23]. Recently three sets of guidelines have been published on LA testing that address the selection of assays, sequence of testing, cutoffs, mixing study technique and interpretation, testing patients on anticoagulant therapy, and interpretive reporting [24]. Although the guidelines have significant overlap and adhere to the basic core of LA diagnosis outlined above and the need for interpretive reporting, they differ in the finer details. Testing patients on anticoagulants with warfarin and unfractionated heparin is generally acceptable because a source of normal plasma and/or a heparin neutralizer are incorporated in the assay. However, this approach is limited to patients with INRs $<3.5$ or receiving unfractionated heparin $<1.0 \mathrm{IU} / \mathrm{ml}$ unless otherwise specified by the manufacturer. For the target specific oral anticoagulants, testing at the trough is suggested if patients cannot be evaluated off drug [23]. The laboratory issues affecting the performance or interpretation of aPL are summarized in Table 3.

Antiphospholipid antibodies as thrombosis risk factors are not all equivalent. For example, higher antibody titer is considered to indicate a higher risk for thrombosis [23]. LA has been shown to be a stronger risk factor than $\mathrm{aCL}$ and anti- $\beta_{2}$ GPI [25-27]. Patients with triple aPL positivity [28] (LA positive, IgG or IgM aCL $>40 \mathrm{GPL}$, IgG or IgM anti$\beta_{2}$ GPI $>99$ th percentile) are at the highest risk for venous and arterial thrombosis and for obstetric complications. In view of these findings, all 3 tests should be performed on the same blood sample, and results should be accompanied by an integrated interpretation with comment [23]. Three risk prediction models have been developed to quantify the risk of thrombosis or obstetrical events in APS [23]. They all aim to assist physicians in stratifying the patient's risk in a similar manor to the prediction models used for cardiovascular disease (i.e., Framingham or Reynolds risk score). While these risk scores may have great potential to support clinical decision making, they still need to be independently and prospectively validated.

Table 3. Laboratory issues affecting the performance or interpretation of aPL.

\begin{tabular}{|l|l|}
\hline \multicolumn{1}{|c|}{ LA } & \multicolumn{1}{c|}{ ACL and anti B2GPI } \\
\hline \hline Failure to prepare platelet poor plasma could mask LA diagnosis & No international standard (calibrator) or units \\
\hline $\begin{array}{l}\text { Different reagents sensitivity to LA } \\
\text { Weak LA may be difficult to diagnose }\end{array}$ & $\begin{array}{l}\text { Positivity related to laboratory established cut-off values - inter-laboratory } \\
\text { variability }\end{array}$ \\
\hline $\begin{array}{l}\text { False positive results from anticoagulants and specific Factor inhibitors } \\
\text { (i.e. FV or FVIII inhibitors) }\end{array}$ & $\begin{array}{l}\text { False positives results with RF, heterophile antibodies, human anti-animal } \\
\text { antibodies, high level of monoclonal immunoglobulins }\end{array}$ \\
\hline
\end{tabular}


A task force in association with the $14^{\text {th }}$ International Congress on Antiphospholipid Antibodies has recently summarized the current data on non-criteria aPL [23]. The review included IgA aPL, tests for antibodies to negatively charged phospholipids and to phosphatidylethanolamine (aPE), antibodies to prothrombin and phosphatidlyserine/ prothrombin complex, and finally antibodies to the domain 1 portion (DI) of $\beta_{2}$ GPI [23]. Some of the more compelling associations reported include IgA anti- $\beta_{2}$ GPI positivity as a risk factor for thrombosis and pregnancy loss in SLE patients; and anti-PS/PT and D1 antibodies as a risk factor for thrombosis. aPhL, a mixture of phospholipids, was also thought to be more specific than aCL in discriminating APS from non-APS [23]. Seronegative APS is the clinical correlate to non-criteria aPL [4]. Patients with seronegative APS have clinical manifestations highly suggestive of APS but are negative for the criteria aPL [4]. Future APS congresses will have to weigh the evidence and decide what, if any, additional aPL to include as new laboratory criteria.

\section{CONFLICT OF INTEREST}

The authors confirm that this article content has no conflict of interest.

\section{ACKNOWLEDGEMENTS}

Declared none.

\section{REFERENCES}

[1] Hughes GR. The antiphospholipid syndrome: ten years on. Lancet 1993; 342(8867): 341-4

[2] Miyakis S, Lockshin MD, Atsumi T, et al. International consensus statement on an update of the classification criteria for definite antiphospholipid syndrome (APS). J Thromb Haemost 2006; 4(2): 295-306.

[3] White RH. The epidemiology of venous thromboembolism. Circulation 2003; 107(23 Suppl 1): I4-I8.

[4] Jayakody AD, Cohen H. Antiphospholipid syndrome. Medicine ; 42(3): 156-61.

[5] Bushnell CD, Goldstein LB. Diagnostic testing for coagulopathies in patients with ischemic stroke. Stroke 2000; 31(12): 3067-78.

[6] Rai RS, Regan L, Clifford K, et al. Antiphospholipid antibodies and beta 2-glycoprotein-I in 500 women with recurrent miscarriage: results of a comprehensive screening approach. Hum Reprod 1995; 10(8): 2001-5.

[7] Mekinian A, Loire-Berson P, Nicaise-Roland P, et al. Outcomes and treatment of obstetrical antiphospholipid syndrome in women with low antiphospholipid antibody levels. J Reprod Immunol 2012; 94(2): 222-6.

[8] Alijotas-Reig J, Ferrer-Oliveras R. The European Registry on Obstetric Antiphospholipid Syndrome (EUROAPS): a preliminary first year report. Lupus 2012; 21(7): 766-8.

[9] Cervera R, Bucciarelli S, Plasin MA, et al. Catastrophic antiphospholipid syndrome (CAPS): descriptive analysis of a series of 280 patients from the "CAPS Registry". J Autoimmun 2009; 32(3-4): 240-5.

[10] Cervera R, Espinosa G, Bucciarelli S, Gomez-Puerta JA, Font J. Lessons from the catastrophic antiphospholipid syndrome (CAPS) registry. Autoimmun Rev 2006; 6(2): 81-4.
[11] Asherson RA, Cervera R, de Groot PG, et al. Catastrophic antiphospholipid syndrome: international consensus statement on classification criteria and treatment guidelines. Lupus 2003; 12(7): 530-4.

[12] de Groot PG, Lutters B, Derksen RH, Lisman T, Meijers JC, Rosendaal FR. Lupus anticoagulants and the risk of a first episode of deep venous thrombosis. J Thromb Haemost 2005; 3(9): 1993-7.

[13] Keeling D, Mackie I, Moore GW, Greer IA, Greaves M. Guidelines on the investigation and management of antiphospholipid syndrome. Br J Haematol 2012; 157(1): 47-58.

[14] Gomez-Puerta JA, Cervera R, Espinosa G, et al. Antiphospholipid antibodies associated with malignancies: clinical and pathological characteristics of 120 patients. Semin Arthritis Rheum 2006; 35(5): 322-32.

[15] Khamashta MA. Hughes Syndrome: History. In: Khamashta MA, Ed. Springer: London 2006; pp. 3-8.

[16] Devreese KM, Pierangeli SS, de LB, Tripodi A, Atsumi T, Ortel TL. Testing for Antiphospholipid antibodies with Solid Phase Assays: guidance from the SSC of the ISTH. J Thromb Haemost 2014; 12(5): 792-5.

[17] Wilson WA, Gharavi AE, Koike T, et al. International consensus statement on preliminary classification criteria for definite antiphospholipid syndrome: report of an international workshop. Arthritis Rheum 1999; 42(7): 1309-11.

[18] Lakos G. Interference in antiphospholipid antibody assays. Semin Thromb Hemost 2012; 38(4): 353-9.

[19] Harris EN, Chan JK, Asherson RA, Aber VR, Gharavi AE, Hughes GR. Thrombosis, recurrent fetal loss, and thrombocytopenia. Predictive value of the anticardiolipin antibody test. Arch Intern Med 1986; 146(11): 2153-6.

[20] Koskela P, Vaarala O, Makitalo R, Palosuo T, Aho K. Significance of false positive syphilis reactions and anticardiolipin antibodies in a nationwide series of pregnant women. J Rheumatol 1988; 15(1): 70-3.

[21] Brandt JT, Triplett DA, Alving B, Scharrer I. Criteria for the diagnosis of lupus anticoagulants: an update. On behalf of the Subcommittee on Lupus Anticoagulant/Antiphospholipid Antibody of the Scientific and Standardisation Committee of the ISTH. Thromb Haemost 1995; 74(4): 1185-90.

[22] Pengo V, Tripodi A, Reber G, et al. Update of the guidelines for lupus anticoagulant detection. Subcommittee on Lupus Anticoagulant/Antiphospholipid Antibody of the Scientific and Standardisation Committee of the International Society on Thrombosis and Haemostasis. J Thromb Haemost 2009; 7(10): 1737-40.

[23] Bertolaccini ML, Amengual O, Andreoli L, et al. 14th international congress on antiphospholipid antibodies task force. Report on antiphospholipid syndrome laboratory diagnostics and trends. Autoimmun Rev 2014; (in press).

[24] Moore GW. Recent guidelines and recommendations for laboratory detection of lupus anticoagulants. Semin Thromb Hemost 2014; 40(2): 163-71.

[25] Galli M, Luciani D, Bertolini G, Barbui T. Lupus anticoagulants are stronger risk factors for thrombosis than anticardiolipin antibodies in the antiphospholipid syndrome: a systematic review of the literature. Blood 2003; 101(5): 1827-32.

[26] Galli M, Luciani D, Bertolini G, Barbui T. Anti-beta 2glycoprotein I, antiprothrombin antibodies, and the risk of thrombosis in the antiphospholipid syndrome. Blood 2003; 102(8): 2717-23.

[27] Opatrny L, David M, Kahn SR, Shrier I, Rey E. Association between antiphospholipid antibodies and recurrent fetal loss in women without autoimmune disease: a metaanalysis. J Rheumatol 2006; 33(11): 2214-21

[28] Pengo V, Banzato A, Bison E, Denas G, Padayattil JS, Ruffatti A Antiphospholipid syndrome: critical analysis of the diagnostic path Lupus 2010; 19(4): 428-31. 